\title{
General equilibrium and the emergence of (non)market clearing trading institutions
}

\author{
Carlos Alós-Ferrer • Georg Kirchsteiger
}

\begin{abstract}
We consider a pure exchange economy, where for each good several trading institutions are available, only one of which is market-clearing. The other feasible trading institutions lead to rationing. To learn on which trading institutions to coordinate, traders follow behavioral rules of thumb that are based on the past performances of the trading institutions. Given the choice of institutions, market outcomes are determined by an equilibrium concept that allows for rationing. We fin that full coordination on the market-clearing institutions without any rationing is a stochastically stable outcome, independently of the characteristics of the alternative available institutions. We also find though, that coordination on certain other, non-market-clearing institutions with rationing can be stochastically stable.
\end{abstract}

Keywords Market clearing · Rationing · Learning in games · General equilibrium

\section{JEL Classificatio $\quad$ C72 $\cdot$ D50}

\section{Introduction}

The theory of general equilibrium is built upon two main assumptions. First, for given prices economic agents behave rationally. Second, prices are determined by the market clearing condition. This paper is part of a research line which attempts to explore to which extent are these assumptions necessary and/or justified

We thank two very careful referees for useful comments which greatly improved the paper.

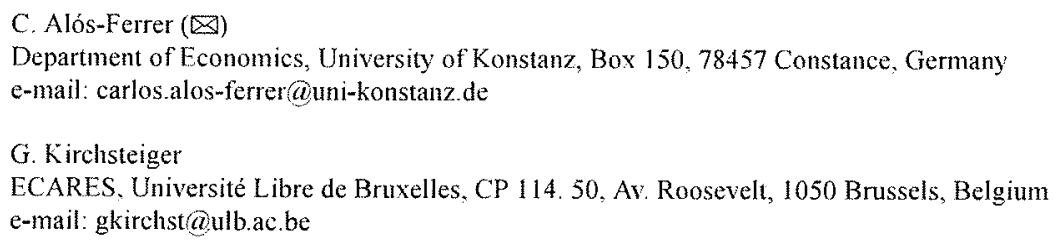


Let us focus on market clearing. While not incorporated into general equilibrium models, actual market exchanges typically take place within institutional frameworks that range from the bazaar to the continuous double auction market. A huge variety of different markets institutions is used, and often different trading institutions exist even for the trade of the same good. Furthermore, empirical and experimental evidence (see, e.g. Holt 1995; Plott 1982; Ockenfels and Roth 2002) shows that the specifi rules of a trading institution affect the transactions' prices and the market outcomes. Often prices are biased toward one market side (see, e.g. Plott (1982) for the experimental investigation of posted-offer institutions) or otherwise constrained (e.g. by price rigidities), so that market-clearing does not always obtain. In this case some market participant face rationing. The question arises whether such an inefficien market institution can survive in the long run, or whether for efficien y reasons it would be replaced by a trading institution that promotes market clearing.

If the market outcomes at alternative market institutions differ, agents face a coordination problem in their institutional choice. A rational trader might opt for an institution that does not lead to market clearing outcomes and does not maximize the gains of trade, simply because all other traders do so and trade at an inefficien institution is still better than having no trading partner at all. Borrowing from the literature of learning in games, we postulate a dynamic learning model for the institutional choice and ask whether traders will learn to coordinate on market-clearing institutions within a general equilibrium framework. When choosing among the trading institutions traders rely on certain behavioral "rules of thumb" for learning. We fin this approach reasonable because due to the complexity of the institution evaluation problem faced by the agents, enriched by the coordination issue, rational learning seems implausible.

In a previous paper (Alós-Ferrer and Kirchsteiger 2008) we posed a similar question within a partial equilibrium buyers-sellers model with a single homogenous good. There we found that, for general learning processes, a market-clearing institution is always active in the long run, independently of the characteristics of the alternative, available institutions. This positive result has to be qualifie by the additional findin that, in general, there might be alternative surviving institutions.' of course, in that model we abstracted from all general equilibrium effects. In particular, the identities of buyers and sellers were a priori fi ed, so that, e.g. a seller could never become a buyer.

In this paper, we account for the general equilibrium feedback effects of the institutional choice. Furthermore, the general equilibrium framework also allows us to endogenize whether a particular trader is a buyer or a seller of a particular good. To do so, we consider a pure exchange economy with $K$ goods where for each good several trading institutions are available. For each good there is always one marketclearing institution. Furthermore, there is a numeraire good for which only one market clearing institution is feasible. At the beginning of the game every trader chooses for

\footnotetext{
1 Multiplicity also arises in Gerber and Betrige (2007), who postulate a finit population of traders with mean-variance utility and a taste for market size who might choose among two identical, market-clearing asset markets. As in Young (1993), traders best-reply to a sample of past play. For a large number of traders, the model predicts that both markets are active and traders split among them.
} 
each good (but the numeraire good) at which market institution he wants to trade this good. Thus, each good can be distinguished according to the institution at which it is traded. After the choices of the institutions, trade is conducted according to the following rule: For each trader, for every good, and for every institution, supply and demand are derived under the additional condition that supply and demand for the goods from the unchosen institutions is zero. This gives us the unrationed supply and demand functions for every trader, good, and institution. This in turn determines the market clearing prices for each good and institution. Non-market-clearing institutions are characterized by a bias function $r$ not identically 1 , which determines the amount of rationing at this institution as a function of the traders actually present. The amount of rationing determines the actual, non-market-clearing price, and the actually realized trades. The model is closed by the residual trade of the numeraire good conducted on its market clearing institution.

The modeling device leading to our equilibrium concept is borrowed from the framework of an economy with rationing as developed by Dreze (1975). Our concept differs from that of Dreze in two respects. First, we directly model rationing, whereas Dreze focused on price rigidities. Second, traders do not take rationing into account when deriving their (unrationed) excess demand function. Rather, the actual demands are determined using the unrationed ones and the rationing schemes. That is, agents do not forecast rationing in advance; rather, the actual realized rationing affects the agents' institution choice in the following period. In other words, the effects of rationing are internalized through learning and not through rational forecasting.

In order to model the learning process we use the stochastic stability techniques brought into the economics literature by Kandori et al. (1993) and Young (1993) to analyze coordination games with players learning on which equilibrium to coordinate. Specifically, we axiomatize the traders' learning process to model the basic idea that they switch from one institution to a different one if they observe that the current-period results (prices and traded quantities as resulting from rationing) are better for them.

Conceptually, our analysis is also related to a recent literature which shows the stability properties of perfectly competitive behavior in learning models with boundedly rational agents. Alós-Ferrer and Ania (2005) show that, in any game where payoffs depend only on the own strategy and an aggregate of all strategies, and those payoffs present strategic substitutability (as, e.g. in Cournot oligopolies or rent-seeking games), the profil where agents maximize payoffs taking the resulting aggregate as given is evolutionarily stable ${ }^{2}$ and the only long-run outcome in imitation-based learning dynamics. Such stability result for aggregate-taking outcomes can be considered as a learning-based foundation for perfectly competitive behavior. The approach of this paper is complementary to the one of Alós-Ferrer and Ania in that we do not consider the stability of outcomes by themselves, but rather the stability of market institutions which channel those outcomes.

As a result, we show that the "Walrasian" constellation, where traders coordinate exclusively on market-clearing institutions (one per good), is always stochastically

\footnotetext{
2 We are referring here to the finite-populatio concept due to Schaffer (1988). In contrast to the infinite-populatio counterpart, an evolutionarily stable strategy in the former sense does not, in general, correspond to a Nash equilibrium.
} 
stable, which could readily be interpreted as a foundation for the market clearing "assumption". We then proceed to show that, in general, certain non-market-clearing institutions also survive, which in turn qualifie the previous interpretation.

We remark up-front that the analysis is kept (relatively) simple by abstracting away from equilibrium multiplicity concerns which arise in nonregular economies. More specifically, we assume the existence of an equilibrium selection correspondence which determines which Walrasian equilibrium with rationing occurs given the traders' institutional choices, and which varies continuously with the institutions' characteristics (in a sense to be made clear below). Alternatively, we could just have assumed enough regularity conditions to ensure equilibrium uniqueness and continuity, but this would just have shifted the focus of the paper.

The paper is structured as follows. In Sect. I we introduce the general equilibrium framework with rationing that we analyze. Then the learning model is introduced. In Sect. 3 we present the main results. Section 4 contains an illustrative example. In Sect. 5 we analyze an extension with agent-specifi rationing parameters which allows us to model institutions with upper or lower bounds on agent's net trades. A discussion of the results and of possible future extensions concludes the paper.

\section{The game form}

There are $N$ traders, $i \in I=\{1, \ldots, N\}$, and $k=1, \ldots, K$ commodities, plus a numeraire commodity $k=0$. Each trader is characterized by excess demand functions

$$
x_{k}^{i}\left(p^{i}\right)
$$

where $p^{i} \in \mathbb{R}_{+}^{K}$ is the price vector with which trader $i$ is confronted. Prices are measured in units of the numeraire good. Since we are interested in learning, there will be an infinit number of trading periods, i.e. the one-shot model is repeated in discrete time. We assume that the excess demand functions of the traders do not change over time. Implicitly we assume that each trader receives the same endowment in each period, and that he has to consume his entire allocation at the end of each period, i.e. after trade has taken place in the period at hand.

We consider a well-behaved economy where equilibrium existence is not an issue and resort to a selection approach to abstract from multiplicity problems. We will be more precise on this below.

For each good $k \neq 0$ there exists a finite nonempty set $Z_{k}$ of institutions at which this good can be traded. In the firs stage of the game, each trader decides for each good the institution at which he wants to trade. Hence, the strategy set of trader $i$ is given by

$$
S^{i}=\prod_{k=1}^{K} Z_{k} .
$$

Given a strategy profil $s \in S=\prod_{i=1}^{N} S^{i}$, denote by $N(s, z)$ the set of players who have chosen to trade good $k \neq 0$ at institution $z \in Z_{k}$. We say that an institution $z$ 
is nonempty given $s$ if $|N(s, z)| \geq 1$, i.e. there are at least one trader at $z$. The set of all nonempty institutions given $s$ is denoted by $A(s)$. The set of nonempty institutions for good $k$ is $A_{k}(s)=A(s) \cap Z_{k}$. An institution $z$ is empty if $N(s, z)=\varnothing$.

Let $Z=\{0\} \cup\left(\cup_{k=1}^{K} Z_{k}\right)$ denote the set of all available institutions, where $z=0$ is the only institution available for good 0 .

We take a parsimonious approach. We will start with a particularly simple subclass of institutions and defin our equilibrium concept for this class only. Then we will generalize the class of considered institutions and defin the corresponding equilibrium concept building on the one introduced for the simpler ones.

A Constant-Rationing Institution $z$ is characterized by a rationing parameter $r_{z}>0$. The functioning of such institutions is as follows. Let $\widetilde{x}_{z}^{i}$ denote the realized excess demand for trader $i$ at institution $z$. This demand is obtained from the unrationed demand and the rationing parameter as follows.

$$
\tilde{x}_{z}^{i}(p, s)= \begin{cases}r_{z} \cdot x_{k}^{i}\left(p^{i}\right) & \text { if } r_{z} \leq 1 \text { and } x_{k}^{i}\left(p^{i}\right) \geq 0, \\ x_{k}^{i}\left(p^{i}\right) & \text { if } r_{z} \leq 1 \text { and } x_{k}^{i}\left(p^{i}\right) \leq 0, \\ \frac{1}{r_{z}} \cdot x_{k}^{i}\left(p^{i}\right) & \text { if } r_{z} \geq 1 \text { and } x_{k}^{i}\left(p^{i}\right) \leq 0, \\ x_{k}^{i}\left(p^{i}\right) & \text { if } r_{z} \geq 1 \text { and } x_{k}^{i}\left(p^{i}\right) \geq 0,\end{cases}
$$

where $p_{k}^{i}=p_{z}$ and $z=z(s, i, k) \in Z_{k}$ is such that $i \in N(s, z)$. We say that an agent $i \in N(s, z)$ is a weak buyer (resp. a weak seller) at institution $z$ if $\widetilde{x}_{z}^{i}(p, s) \geq 0$ (resp. $\left.\tilde{x}_{z}^{i}(p, s) \leq 0\right)$. The realized excess demand for the numeraire is given by

$$
\widetilde{x}_{0}^{i}(p, s)=-\sum_{k=1}^{K} p_{z(s, i, k)} \widetilde{x}_{z(s, i, k)}^{i}(p, s) .
$$

The interpretation is as follows. At every institution $z$ for commodity $k$ where a trader is active, he wants to trade $x_{k}^{i}\left(p^{i}\right)$. There are, however, institutions where one market side is rationed. If $r_{z}<1$, buyers are rationed and can realize only $r_{z}$ times their intended trades, whereas sellers face no restriction. If $r_{z}>1$, sellers can only realize $\frac{1}{r_{z}}$ of their intended trades. If $r_{z}=1$, neither market side is rationed and we say that institution $z$ is fully competitive. We assume that, for each good $k$ there is at least one fully competitive, Walrasian institution $w_{k} \in Z_{k}$ such that $r_{w_{k}}=1$. Commodity 0 is used as the medium of exchange at all institutions for all other commodities and, therefore, there is no rationing for this commodity. The interpretation is that residual trade is conducted with the numeraire good in its market-clearing institution. This closes the model. ${ }^{3}$

In equilibrium, prices are determined in such a way that markets "clear" taking rationing into account.

Definitio 1 Given a vector $r=\left(r_{z}\right)_{z \in Z}$ and a strategy profil $s$, an $(r, s)$-equilibrium is given by a price vector $p^{*}=\left(p_{z}^{*}\right)_{z \in Z}$ such that, for all $k \neq 0$ and for all $z \in Z_{k}$,

3 This idea of a numeraire good which is traded without rationing is taken from Dreze (1975). 
(i) $\sum_{i \in N(s, z)} \widetilde{x}_{z}^{i}\left(p^{*}, s\right)=0$,

(ii) $\sum_{i=1, \ldots, N} \widetilde{x}_{0}^{i}\left(p^{*}, s\right)=0$.

We turn now to our regularity assumption. Note that an $(r, s)$-equilibrium is just an equilibrium of an expanded economy where the goods of different nonempty institutions are regarded as different goods (independently of whether they are physically the same) and excess demands are zero for goods traded at an institution a trader is not present at. Thus, standard assumptions on the individual excess demand functions will yield existence of $(r, s)$-equilibria with strictly positive equilibrium prices at every nonempty institution. We will be left with a potential multiplicity problem (unless we assume gross substitutability among all goods). In order to obtain well-define and continuous payoff functions, we will simply assume a continuous selection from the Walrasian equilibrium correspondence.

(A0): Given a set of institutions $Z$ and a fi ed profil $s \in S$, there exists a continuous selection of the $(r, s)$-equilibrium correspondence; that is, the equilibrium price $p(r)$ as a function of the institutional bias $r=\left(r_{z}\right)_{z \in Z}$ is continuous.

For Theorem 1, continuity is actually not necessary; all we need is that the selected equilibrium is well-define (in order to be able to defin the game of institution choice). Continuity is required for Theorem 2; actually, it is enough if the selection is continuous (in the institutional biases $r$ ) on an open set containing $(1, \ldots, 1)$. This is essentially a regularity assumption which is fulfille generically under standard conditions; in particular it would be fulfille if gross substitutability is assumed in order to ensure equilibrium uniqueness. ${ }^{\frac{4}{4}}$ In other words, once players make their institution choices, (Walrasian) prices are realized within each institution, and we use a continuous selection to abstract from multiplicity issues.

Remark 1 The continuous-selection approach we introduce here is similar to (and has been inspired by) the continuous random selection technique introduced by Allen (1985) (see also Mas-Colell and Nachbar 1991; Faias et al. 2002; see also Stahn 1999 for an application in a different context), with the crucial difference that, rather than a probability measure over equilibria, our selection directly specifie an equilibrium. This is practicable in our case because continuity refers to the vector of rationing parameters and not to individual characteristics of the agents. That is, the excess demand functions are kept fi ed, and continuity is only required for variations in the constant-bias parameters. The analysis is hence simpler than the one in the case of continuous random selections. Still, it is conceivable that, for instance, for a particular economy the bias vector $r=(1, \ldots, 1)$ represents a singular point and no continuous selection can be specified It is such cases which are excluded by our assumption.

\footnotetext{
${ }^{4}$ A possible set of conditions is as follows: (i) $x_{k}^{i}\left(p^{i}\right)$ is differentiable and strictly decreasing in $p_{k}^{i}$; (ii) no trader can sell short, i.e. there exists an $a>0$ such that for all $p^{i} \in \mathbb{R}_{+}^{K}, x_{k}^{i}\left(p^{i}\right)>-a$, (iii) each trader is endowed with a positive amount of every good, i.e. there exists a $p^{i} \in \mathbb{R}_{+}^{K}$, such that $x_{k}^{i}\left(p^{i}\right)<0$; and (iv) if $p^{i n} \rightarrow p^{i}$ where $p^{i} \neq 0$ and $p_{k}^{i}=0$, then $x_{k}^{i}\left(p^{i n}\right) \rightarrow \infty$ (this is fulfille whenever the underlying preferences are strongly monotone). Adding assumptions on gross substitutability of the goods would also ensure uniqueness; in this case, continuity would follow immediately by definition of $(r, s)$-equilibrium.
} 
We now generalize the class of considered institutions as follows. A VariableRationing Institution is characterized by a function

$$
r_{z}: \mathcal{P}(I) \backslash\{\varnothing\} \longrightarrow \mathbb{R}_{++}
$$

which assigns a rationing parameter $r_{z}(J)$ to each possible, nonempty set $J \subseteq I$ of agents actually trading at $z$. Of course, the function might be constant, hence constantrationing institutions, including perfectly competitive ones, form a subset of the set of variable-rationing institutions. Denote $r_{z}(s)=r_{z}(N(s, z))$ for all $z \in A(s)$. The equilibrium concept is then adapted as follows. ${ }^{5}$

Definitio 2 Given a family of variable-rationing institutions $Z$, each characterized by a rationing function $r_{z}$, and given a strategy profil $s$, an $s$-equilibrium is an $(r(s), s)$ equilibrium, where $r(s)=\left(r_{z}(s)\right)_{z \in Z}$.

From now on, we will consider variable-rationing institutions, although constant-rationing institutions are of course a particular case.

The described model corresponds to a game form where strategies model the choice of institutions, and outcomes correspond to actual allocations of goods among traders. If demands are derived from maximization of standard utility functions, a natural way to complete this game form to a game would be simply to take payoffs to be given by the corresponding utilities derived from the allocations. We decided, however, to use a more general approach in order to allow for nonmaximizing behavior.

Remark 2 Agents play two roles in our model, as boundedly rational players in a game of institution selection, and as traders in the general equilibrium model which results once institutions have been chosen. A crucial assumption of the model (which we will make explicit in the next section) is that institution choice is based on past, observed outcomes, and the institution's characteristics affect institution choice through learning only. In particular, agents will not plan to, e.g. manipulate their excess demand functions when planning an institution choice. Obviously, for any given rationing scheme, traders might have an incentive to misrepresent their true excess demand functions. While the manipulation of demand is potentially very important for the understanding of the functioning of markets, this problem is not restricted to our rationing framework. In fact, if traders are explicitly modeled as agents who state their demand functions and have the possibility of untruthfully reporting them, they would also have an incentive to do so in a standard Arrow-Debreu framework. ${ }^{6}$ Since we are mainly interested in the choice of the trading platforms, we do not take the possibility of manipulating demand into account. There are two simple ways to think about the structure of the resulting model. The firs is to explicitly consider boundedly rational agents, who move from one institution to another following observable, past results, but do not

\footnotetext{
5 The analysis and the results remain unclianged if we directly defin $r_{z}$ on $S$, which would allow the rationing parameter to depend, e.g. on the set of traders present at other institutions. However, we fin the additional examples we would be able to cover through this extra generality to be rather artificial

${ }^{6}$ For instance, traders could strategically withhold part of their endowments, thereby creating artificia scarcities. This possibility is analyzed in Poslewaite (1979), Yi (1991), and Moreno-Garcia (2006).
} 
anticipate future ones, and in particular forgo manipulation opportunities. The second is to consider market institutions as "black-box mechanisms" which generate a market result given the true characteristics (excess demand functions) of traders in them. That is, if agents, e.g. do attempt to fake their demands in order to manipulate the result, the outcome delivered by the trading institution already internalizes the effect of attempted manipulations. Although this latter interpretation is problematic for constant-rationing institutions, for variable-rationing institutions the function $r_{z}$ just discussed could potentially incorporate Nash-equilibrium considerations for a market mechanism with demand manipulation. An explicit modeling of this possibility is beyond the scope of this paper.

\section{The learning model}

Behavior of traders will be modeled through two elements. First, whether a trader is able to revise his choice at all in a given period, and second, how will he choose the institutions he wants to trade at if given the opportunity to revise his previous choice. The revision probabilities will be described in Sect. 3.2.

\subsection{The choice of institutions}

How choices are revised is captured by behavioral rules, which can in general be expressed as mappings

$$
B^{i}: S \rightarrow \triangle S^{i}
$$

i.e. given that the current strategy profil is given by $s^{\prime}, B^{i}\left(s^{\prime}\right)\left(s^{i}\right)$ denotes the probability that trader $i$ will choose the combination of institutions prescribed in $s^{i}$ next period, for any arbitrary $s^{i}$. Note that we allow each trader to correlate institution choices for different goods. Given an institution $z \in Z_{k}$, denote further by $B_{k}^{i}\left(s^{\prime}\right)(z)$ the probability that trader $i$ will choose institution $z$ for good $k$ next period. That is, $B_{k}^{i}$ is the marginal probability distribution derived from $B^{i}$, but $B^{i}$ need not be equal to the product of its marginal distributions.

We assume that traders receive or make use of far less information than that contained in the whole strategy profil $s$. Specifically, they observe, for every nonempty institution, only the price and the rationing parameter. Formally, denote by

$$
I(s)=\left[A(s),\left(p_{z}(s), r_{z}(s)\right)_{z \in A(s)}\right]
$$

the information available to traders. We assume that

$$
B^{i}\left(s_{1}\right)=B^{i}\left(s_{2}\right) \quad \text { whenever } s_{1}^{i}=s_{2}^{i} \text { and } I\left(s_{1}\right)=I\left(s_{2}\right)
$$

This means that, when taking a decision, traders only take their previous decision, prices and rationing of nonempty institutions into account. 
Example 1 A particularly simple class of behavioral rules results if traders take into account only the actual prices in nonempty institutions, and which market side is rationed (if any), but not the exact amount of rationing. Formally, a behavioral rule $B^{i}$ fulfill this condition if

$$
B^{i}\left(s_{1}\right)=B^{i}\left(s_{2}\right) \quad \text { whenever } s_{1}^{i}=s_{2}^{i} \text { and } I^{E}\left(s_{1}\right)=I^{E}\left(s_{2}\right),
$$

where

$$
I^{E}(s)=\left[A(s),\left(p_{z}(s), \operatorname{sign}\left(r_{z}-1\right)\right)_{z \in A(s)}\right]
$$

is the information traders actually use.

We say that an agent $i \in N(s, z)$ leaves institution $z$ with positive probability if the probability that he stays at institution $z$ is strictly below I, i.e.

$$
B_{k}^{i}(s)(z)<1 .
$$

We now spell out our assumptions on the behavioral rules of traders:

(A1) For every strategy profil $s$, every good $k$, and every institution $z \in A_{k}(s)$,

(i) if $i \in N(s, z)$ with $\widetilde{x}_{k}^{i}(s) \geq 0$ and there exists $z^{\prime} \in A_{k}(s)$ with $r_{z^{\prime}}(s) \geq$ $r_{z}(s)$ (i.e. buyers are more rationed at $z$ than at $z^{\prime}$, if at all rationed) and $p_{z^{\prime}}(s) \leq p_{z}(s)$, or

(ii) if $i \in N(s, z)$ with $\widetilde{x}_{k}^{i}(s) \leq 0$ and there exists $z^{\prime} \in A_{k}(s)$ with $r_{z^{\prime}}(s) \leq$ $r_{z}(s)$ (i.e. sellers are more rationed at $z$ than at $z^{\prime}$, if at all rationed) and $p_{z^{\prime}}(s) \geq p_{z}(s)$

then agent $i$ leaves institution $z$ with positive probability.

The intuition for Al is straightforward. Suppose the excess demand of a buyer of good $k$ is weakly decreasing in the price of that good (such an assumption will be typically made in order to build economies which satisfy A0). The buyer trades this good at a given institution $z$. He observes that buyers at another institution $z^{\prime}$ for good $k$ are less rationed (or not rationed at either institution) and pay a strictly lower price. Of course, a myopic buyer will not expect to become a seller if he switches to $z^{\prime}$. Consequently, the buyer wants to switch either to $z^{\prime}$ with at least some probability, or to some other (maybe even better) third institution. Hence, he does not want to stay with certainty at $z$. This is precisely what Al states. The same intuition holds for a seller if he observes a higher price at another institution where sellers are less rationed.

One particular implication of Al is that, if a trader is confronted with the same rationing in two different institutions (e.g. if he is not rationed in either institution), he does not stay for sure at the institution with the worse price.

We want to extend the reasoning above to institutions where the trader is not too much rationed. Take a situation where a trader at institution $z$ is not rationed. $\mathrm{He}$ observes another institution $z^{\prime}$ where he would received a better price, but at the cost of some rationing. Provided that the rationing is moderate compared to the price difference, he wants to switch. This reasoning is formalized by the following assumption: 
(A1 $\left.{ }^{*}\right)$ Fix a strategy profil $s$, a good $k$, and an institution $z \in A_{k}(s)$. For each price $p^{\prime}<p_{z}(s)$ and each price $p^{\prime \prime}>p_{z}(s)$, there exist $\underline{r}\left(p^{\prime}, p_{z}(s)\right)<1$ and $\bar{r}\left(p^{\prime \prime}, p_{z}(s)\right)>1$ such that agent $i \in N(s, z)$ leaves institution $z$ with positive probability if either

(i) $\widetilde{x}_{k}^{i}(s) \geq 0, r_{z}(s) \geq 1$, and there exists $z^{\prime} \in A_{k}(s)$ with $p_{z^{\prime}}(s) \leq p^{\prime}$ and $r_{z^{\prime}}(s) \geq \underline{r}\left(p^{\prime}, p_{z}(s)\right)$, or

(ii) $\widetilde{x}_{k}^{i}(s) \leq 0, r_{z}(s) \leq 1$ and there exists $z^{\prime \prime} \in A_{k}(s)$ with $p_{z^{\prime}}(s) \geq p^{\prime \prime}$ and $r_{z^{\prime \prime}}(s) \leq \bar{r}\left(p^{\prime \prime}, p_{z}(s)\right)$.

Under the assumptions above, we obtain the following result for general behavioral rules:

Lemma 1 Assume Al. Given any strategy profile $s \in S$ such that, for a good $k$, both the institution $w_{k}$ and another, not fully competitive $z \in Z_{k}$ are nonempty, either all weak buyers or all weak sellers $i \in N(s, z)$ leave $z$ with positive probability.

Proof Suppose $r_{z}(s)<1$. This implies that, at $z$, weak sellers are not rationed. If sellers wants to leave institution $z$, the proof is completed. If some seller wants to stay at institution $z$ with certainty, then by A1(ii) it follows that $p_{w_{k}}(s)<p_{z}(s)$. Since buyers are rationed at institution $z$ but there is no rationing at $w_{k}$, A 1(i) implies that all weak buyers have positive probability to leave institution $z$. The proof for $r_{z}(s)>1$ is analogous.

(A2) For every strategy profil $s$, every good $k$, and every two institutions $z, z^{\prime} \in Z_{k}$, we have that, if $z$ is nonempty and $z^{\prime}$ is empty under $s$, then

$$
B_{k}^{j}(s)\left(z^{\prime}\right)=0 \quad \text { for all } j \in N(s, z)
$$

Intuitively, if the demand function of a trader prescribes to trade in a given, nonempty institution, it is because the trader prefers trading over no trading. Hence, a switch from an nonempty institution (where he is allowed to trade to at least some extent) to an empty institution (where he will not be able to trade) will never benefi him and therefore he will never switch to that empty institution. Alternatively, this assumption can be interpreted as an information constraint: empty institutions are not even observed, hence they are not perceived as alternatives.

Example 2 Consider the following simple behavioral rule. Excess demand functions are derived from monotonic, continuous utility functions $u^{i}$ and fi ed initial endowments. Fix an agent $i$ and a good $k$, traded by $i$ at institution $z$. Denote the actual consumption bundle of agent $i$ by $q^{i}=\left(q_{\ell}^{i}\right)_{\ell=0}^{K}$. For each alternative, nonempty institution $z^{\prime} \in Z_{k}$, let $q_{0}^{i}\left(z^{\prime}\right)$ and $q_{k}^{i}\left(z^{\prime}\right)$ be the solution of the agent's optimization problem when he is endowed with $q^{i}$, can only change his consumption of goods 0 and $k$, and the price of good $k$ is $p\left(z^{\prime}\right)$. Let $\widetilde{q}_{0}^{i}\left(z^{\prime}\right)$ and $\widetilde{q}_{k}^{i}\left(z^{\prime}\right)$ be the quantities after rationing at institution $z^{\prime}$ is taken into account. Agent $i$ will randomize among all $z^{\prime} \in A_{k}(s)$ in

$$
\arg \max _{z^{\prime} \in A_{k(s)}} u^{i}\left(\widetilde{q}_{0}^{i}\left(z^{\prime}\right), q_{1}^{i}, \ldots, \widetilde{q}_{k}^{i}\left(z^{\prime}\right), \ldots, q_{K}^{i}\right)
$$


Assumptions (A1), ( $\left.1^{*}\right)$, and (A2) are immediately satisfied Such a rule is myopic in three respects. First, agents do not take into account the fact that switching from one institution to another affects the market outcome. Second, in making such simple, virtual utility comparisons, agents neglect the feedback effects that changes in the market outcome for one good has in the outcome (and price) for other goods. Third, agents also neglect the fact that, if actually faced with the prices observed at other institutions, they might want to substitute with other goods.

\subsection{Revision opportunities}

When can agents revise their choices? It is common in learning models to explicitly introduce some inertia allowing for the possibility that not all agents are able to revise strategies simultaneously. Different specification of how revision opportunities arrive give rise to different dynamics and often affect the results. Rather than adopting a specifi formulation, here, we postulate a general class of dynamics encompassing the standard examples (and many others), which are then reviewed below. See Alos-Ferrer (2003) or Alos-Ferrer and Netzer (2007) for a discussion.

Let $E(i, s)$ denote the event that agent $i$ receives revision opportunity when the current state is $s$, and let $E^{*}(i, s) \subseteq E(i, s)$ denote the event that agent $i$ is the only agent receiving revision opportunity in $s$.

Assumption D: $\operatorname{Pr}\left(E^{*}(i, s)\right)>0$ for every agent $i$ and state $s$.

Assumption D corresponds to regularity of a revision process in Alós-Ferrer and Netzer (2007). It implies that $\operatorname{Pr}(E(i, s))>0$, i.e. every agent has strictly positive probability of being able to revise at any given state.

Assumption D is rather general. It is fulfille by the standard models considered in the literature of learning in games. In these models, revision opportunities are either modeled through independent probabilities (a case we call independent inertia) or assumed to arrive in an asynchronous way (also called Asynchronous learning).

Independent Inertia. There is an exogenous, independent (across traders and periods) probability $0<1-\rho<1$ such that the agent does not get revision opportunity in a given state (inertia). Obviously, $\operatorname{Pr}\left(E^{*}(i, s)\right)=\rho(1-\rho)^{N-1}>0$ for any agent $i$, hence verifying $\mathrm{D}$.

Asynchronous Learning. Each period, only one agent (i.e. either a buyer or a seller) is (randomly) selected and allowed to revise his strategy. Hence, $\operatorname{Pr}\left(E^{*}(i, s)\right)=\frac{1}{N}$ for any trader $i$, verifying $\mathrm{D}$.

The specificatio above allows for more general learning processes than those described by independent inertia or asynchronous learning. Since the revision probability $\operatorname{Pr}(E(i, s))$ is a function of the state $s$, it might depend, e.g. on the difference of payoffs between different institutions (so that unsatisfie traders are more likely to revise), or on idiosyncratic characteristics of the currently chosen institution.

\subsection{Learning dynamics}

Assumption D and the behavioral rules $B^{i}$ defin a stationary Markov chain on the (finite state space $S$. Given two states $s, s^{\prime} \in S$, denote by $P\left(s, s^{\prime}\right)$ the probability of 
transition from $s$ to $s^{\prime}$ in one period for the learning process. The transition matrix of the process is given by $P=\left[P\left(s, s^{\prime}\right)\right]_{s, s^{\prime} \in S}$.

Before we analyze the learning dynamics, we summarize the basic results on Markov chains (see, e.g. Feller 1968 or Karlin and Taylor 1975 for further details). An absorbing set is a minimal subset of states which, once entered, is never abandoned. An absorbing state is an element which forms a singleton absorbing set, i.e. a state $s$ such that $P(s, s)=1$. An invariant distribution is a distribution over states $\mu \in \Delta(S)$ which, if taken as initial condition, is reproduced in probabilistic terms after updating (more precisely, $\mu \cdot P=\mu$ ). Every absorbing set $A \subseteq S$ induces a unique invariant distribution $\mu_{A}$ with support $A$. The set of all invariant distributions of the process is the convex hull of the invariant distributions associated to the absorbing sets. By the Ergodic Theorem, $\mu_{A}$ describes the time-average behavior of the system once (and if) it gets into the absorbing set $A$. That is, $\mu(s)$ is the limit of the average time that the system spends in state $s$, along any sample path that eventually gets into $A$. If, additionally, the absorbing set $A$ is aperiodic ${ }^{7}$ then $\mu_{A}$ describes also the long-run probabilities of the states in the class, $\lim _{T \rightarrow \infty} \eta \cdot P^{T}=\mu_{A}$ for all probability distributions $\eta$ whose support is contained in $A$. This result is called the Fundamental Theorem of Markov Chains.

A Markov chain is ergodic if it has a unique absorbing set. The (unique) invariant distribution constitutes the long-run prediction for such a process, since it represents the limit behavior of the process independently of initial conditions. If the process is not ergodic, then several invariant distributions exist, describing the long-run behavior along different sample paths, i.e. the prediction depends on the initial conditions.

Obviously, by A2, full coordination at exactly one institution per good is an absorbing state. Hence, there is a multiplicity of absorbing sets. In order to select among them, and following the literature, we proceed to study stochastic stability. The dynamics is enriched with a perturbation in the form of experiments (or mistakes) as follows. With an independent probability $\varepsilon>0$, each trader, in each round, might experiment (or make a mistake or mutate), and simply pick a new combination of institutions at random, ${ }^{8}$ independently of other considerations.

The dynamics with experimentation is called perturbed learning process. Its transition matrix is denoted by $P^{\varepsilon}$. Since experiments make transitions between any two states possible, the perturbed process has a single absorbing set formed by the whole state space (such processes are called irreducible). Hence, the perturbed process is ergodic. The corresponding (unique) invariant distribution is denoted $\mu(\varepsilon)$. The limit invariant distribution (as the rate of experimentation tends to zero) $\mu^{*}=\lim _{\varepsilon \rightarrow 0} \mu(\varepsilon)$ exists and is an invariant distribution of the unperturbed process $P^{0}=P$ (see, e.g. Kandori et al. 1993; Young 1993 or Ellison 2000).

The limit invariant distribution singles out a stable prediction of the unperturbed dynamics, in the sense that, for any $\varepsilon>0$ small enough, the play approximates that

\footnotetext{
7 Loosely speaking, an absorbing set is aperiodic if it contains no deterministic nontrivial cycles. A sufficien condition is that $P(s, s)>0$ for some state $s$ in the set. Any absorbing state is aperiodic.

8 We mean that a combination of institutions is picked up according to a pre-specifie probability distribution having full support, for instance uniformly. The exact distribution does not affect the results, as long as it has full support, and does not depend on $\varepsilon$.
} 
described by $\mu^{*}$ in the long run. The states in the support of $\mu^{*}$, i.e. $\left\{s \in S \mid \mu^{*}(s)>0\right\}$ are called stochastically stable states or long-run equilibria. Clearly, the set of stochastically stable states is the union of some absorbing sets of the original, unperturbed chain $(\varepsilon=0)$.

In the sequel, whenever we say absorbing sets or states, we refer to the dynamics without experimentation. Since the perturbed dynamics is irreducible, no confusion should arise.

\subsection{Radius and coradius of a state}

Given two absorbing sets $X$ and $Y$, let $c(X, Y)>0$ (referred to as the transition cost from $X$ to $Y$ ) denote the minimal number of mistakes necessary for a direct transition from $X$ to $Y$, i.e. a positive probability path starting in an element of $X$ and leading to an element in $Y$, which does not go through any other absorbing set.

Transitions need not be direct, though. Defin a path from $X$ to $Y$ as a finit sequence of absorbing sets $\Lambda=\left\{X=S_{0}, \ldots, S_{K}=Y\right\}$. Let $S(X, Y)$ be the set of paths from $X$ to $Y$. Given a path $\Lambda$, defin its length $l(\Lambda)$ as the number of elements of the sequence minus 1 , so that $\Lambda=\left\{X=S_{0}, \ldots, S_{l(\Lambda)}=Y\right\}$. We extend the cost function to paths by $c(\Lambda)=\sum_{k=1}^{l(\Lambda)} c\left(S_{k-1}, S_{k}\right)$.

Now, defin $C(X, Y)=\min _{\Lambda \in S(X, Y)} c(\Lambda)$ to be the minimal number of mistakes required for a (possibly indirect) transition from $X$ to $Y$.

The Radius of an absorbing set $X$ is define as

$$
R(X)=\min \{C(X, Y) \mid Y \text { is an absorbing set, } Y \neq X\},
$$

i.e. the minimal number of mistakes needed to leave $X$ toward another absorbing set.

Intuitively, the radius measures how easy it is to destabilize an absorbing set. To obtain a measure for the accessibility of an absorbing set, we defin the coradius of $X$ as

$$
C R(X)=\max \{C(Y, X) \mid Y \text { is an absorbing set, } Y \neq X\} .
$$

Ellison (2000) provides a powerful result which states that, if $R(X)>C R(X)$ for a given absorbing set $X$, then $X$ is the unique stochastically stable set. The intuition is clear, for the inequality $R(X)>C R(X)$ simply expresses the idea that $X$ is easier to reach than to leave. This result, though, is not enough for our purposes. We will make use of a fine result which makes use of the concept of modifie coradius. This concept takes into account that paths from $Y$ toward $X$ which pass through other absorbing sets have an increased probability which can be measured exactly by the radius of the intermediate absorbing sets.

Given a path $\Lambda=\left\{S_{0}, \ldots, S_{l(\Lambda)}\right\}$, defin the modifie transition costs $c^{*}(\Lambda)=$ $c(\Lambda)-\sum_{k=1}^{l(\Lambda)-1} R\left(S_{k}\right)$. Defin accordingly $C^{*}(X, Y)=\min _{\Lambda \in S(X, Y)} c^{*}(\Lambda)$. Then, the modifie coradius is given by

$$
C R^{*}(X)=\max \left\{C^{*}(Y, X) \mid Y \text { is an absorbing set, } Y \neq X\right\} \text {. }
$$


The following Lemma, adapted from Ellison (2000), states that, whenever the radius exceeds the (modified coradius, the absorbing set is easier to reach than to destabilize, and hence it is stochastically stable.

\section{Lemma 2 Let $A$ be an absorbing set. Then:}

(i) If $R(A) \geq C R^{*}(A)$, the states in $A$ are stochastically stable.

(ii) If $R(A)>C R^{*}(A)$, the only stochastically stable states are those in $A$.

(iii) If the states in an absorbing set $B$ are stochastically stable and $R(A)=C^{*}$ $(B, A)$, the states in $A$ are also stochastically stable.

Proof Part (ii) follows immediately from Ellison (2000, Theorem 2). Part (iii) follows from Ellison (2000, Theorem 3). Part (i) reduces to (ii) if the inequality is strict. If $R(A)=C R^{*}(A)$, let $B$ be any absorbing set containing stochastically stable states (which always exist). By definition of Coradius, $C R^{*}(A) \geq C^{*}(B, A)$. If this inequality is strict, it follows from Ellison (2000, Theorem 3 ) that $B$ does not contain any stochastically stable state, a contradiction. Hence $R(A)=C R^{*}(A)=C^{*}(B, A)$ and the conclusion follows from part (iii).

\section{Stochastic stability}

We now turn to the analysis of the stochastically stable states. We will show that, for an arbitrary number of traders, goods, and feasible institutions, full coordination on a Walrasian institution for each good is stochastically stable. It turns out, though, that full coordination on certain non-market-clearing institutions is also stochastically stable.

\subsection{Stability of Walrasian institutions}

The Walrasian state $W$ is the state where, for each good $k$, all traders coordinate in the corresponding fully competitive institution $w_{k}$. That is, $A(W)=\left\{w_{k}\right\}_{k=1}^{K}$.

Theorem 1 Under $A 1, A 2$, and $D, W$ is stochastically stable.

Proof By A2, the Walrasian state $W$ is an absorbing state. We will show that, for any state $s$, there exists a chain of single mutations connecting $s$ with $W$. Hence, the modifie coradius of $W$ is equal to 1 .

Start at state $s$, and consider a good $k$ such that there is some nonempty institution $z$ for $k$ other than $w_{k}$. With a single mutation, some agent will choose institution $w_{k}$. By Lemma 1 and Assumption $\mathrm{D}$, with positive probability one agent who was previously trading good $k$ at $z$ will leave institution $z$. In the resulting state, Lemma I and Assumption D again ensure that another agent (if any agent is left) will leave $z$. Iteration of this argument ends with a state where institution $z$ becomes empty. Repetition of the argument for all other nonempty institutions for good $k$ ends with a state $s^{\prime}$ where the only nonempty institution for good $k$ is $w_{k}$. Note that, by $\mathrm{A} 2$, none of the empty institutions can become nonempty in the process. 
This new state $s^{\prime}$ might be in some absorbing set or not. If not, there exists a positive probability path, involving no further mutations, connecting $s^{\prime}$ to a new state $s^{\prime \prime}$ in some absorbing set. By A2, the only nonempty institution for good $k$ in $s^{\prime \prime}$ is also $w_{k}$.

From state $s^{\prime \prime}$, the same argument (involving a single mutation) allows to reach a new state where the only nonempty institution for another good $k^{\prime}$ is $w_{k^{\prime}}$. Repetition of this arguments leads to the state $W$.

Since the radius of all intermediate absorbing sets is 1 and they are left with a single mutation, the modifie coradius of $W$ is 1 . Since the radius of $W$ is at least 1 , it follows from Lemma 2 that $W$ is stochastically stable.

The stability of Walrasian institutions shown in Theorem 1 is a powerful result. Actually, the argument can be used to show that no other institution can coexist with the Walrasian ones in a stochastically stable state.

Corollary 1 Assume A1, A2, and D. Let s be a state where, for some good $k$, the fully competitive institution $w_{k}$ and another institution $z$ are simultaneously nonempty. Then, $s$ cannot be in any absorbing set, and hence cannot be stochastically stable.

Proof At state $s$, by Lemma I and Assumption D, with positive probability one of the agents trading good $k$ at $z$ will leave institution $z$. Iterating the argument as in the proof of Theorem 1 leads to a state where institution $z$ becomes empty. But, by assumption $A 2$, the mutation-free dynamics can never lead back to a state where $z$ is nonempty. Hence there exists a positive-probability path from $z$ to a state from which it is not possible to return, implying that $z$ does not belong to an absorbing set.

Still, we will now show that alternative trading institutions can be stochastically stable, and hence be observed a significant fraction of the time in the long run.

\subsection{Stability of other institutions}

In this section, we will show that institutions other than the Walrasian ones can also be stochastically stable. In order to make this point, we can restrict ourselves to the simplest possible institutions, namely those with constant rationing. We will also exclude from the analysis certain knife-edge economies.

Definitio 3 An economy $\left(x_{k}^{i}\right)_{k=1, \ldots K}^{i=1, \ldots N}$ is nondegenerate if whenever the institution set $Z$ is such that two alternative market-clearing institutions for the same good $k$ are available, $w_{k}$ and $w_{k}^{\prime}$, then for any strategy profil $s$ it holds that $p_{s}\left(w_{k}\right) \neq p_{s}\left(w_{k}^{\prime}\right)$.

Nondegeneracy is a very weak condition. For example, suppose that excess demand functions are derived from underlying utilities and endowments. Since for any strategy profil $s$ the sets of agents at the two institutions $w_{k}$ and $w_{k}^{\prime}$ are disjoint, generically in endowments the prices will always be different in these two institutions.

Theorem 2 Assume A1,A1*,A2, and $D$. For nondegenerate economies, there exist $\underline{r}_{k}<1$ and $\bar{r}_{k}>1$ for all $k$ such that, if $z_{k}\left(r_{k}\right)$ is an institution for good $k$ with constant rationing parameter $\left.r_{k} \in\right] \underline{r}_{k}, \bar{r}_{k}[$, the state $\omega$ where all traders coordinate at the institutions $z_{k}\left(r_{k}\right)$ for all $k$ is stochastically stable. 
Proof Recall that, by A0, and given a fi ed strategy profil $s$, the equilibrium price vector $p(r)$ is continuous in $r=\left(r_{1}, \ldots, r_{K}\right)$.

Since $W$ is stochastically stable, it suffice to show that there exists a positive probability transition from $W$ to the new state which involves only chains of single mutations (hence the modifie coradius of the new state is 1).

We start at state $W$ and proceed iteratively. At each step, we will show that one mutation suffice for all traders to move away from $w_{k}$ and fully coordinate at $z_{k}\left(r_{k}\right)$.

Take good 1. Let $S_{1}$ be the set of strategy profile $s$ such that $\left.A(s) \cap Z_{1}=\left\{w_{1}, z_{1}\right)\right\}$ and $A(s) \cap Z_{k}=\left\{w_{k}\right\}$ for all $k \neq 1$.

Given $s \in S_{1}$ and $z_{1}=z_{1}(1)$, let $\varepsilon_{s}^{1}=\left|p_{s}\left(z_{1}(1)\right)-p_{s}\left(w_{1}\right)\right|$ where $p_{s}\left(z_{1}(1)\right)$ and $p_{s}\left(w_{1}\right)$ are the equilibrium prices at the two nonempty institutions for good 1 (which are actually both fully competitive). Let

$$
0<\delta<\frac{1}{2} \min \left\{\varepsilon_{s}^{1} \mid s \in S, \varepsilon_{s}^{1}>0\right\} .
$$

By continuity, there exist $\underline{r}_{1}^{\prime}<1$ and $\bar{r}_{1}^{\prime}>1$ such that, for every $\underline{r}_{1}^{\prime}<r_{1}<\bar{r}_{1}^{\prime}$, $\left|p_{s}\left(z_{1}\left(r_{1}\right)\right)-p_{s}\left(z_{1}(1)\right)\right|<\delta$ for all $s \in S_{1}$, where $p_{s}\left(z_{1}(1)\right)$ is the equilibrium price when $z_{1}=z_{1}(1)$ and $p_{s}\left(z_{1}\left(r_{1}\right)\right)$ is the equilibrium price when $z_{1}=z_{1}\left(r_{1}\right)$.

We want to show that, for any $s \in S_{1}$, and for $r_{1}$ close enough to 1 , there always exists a trader at $w_{1}$ who wants to change to $z_{1}\left(r_{1}\right)$. Since there are no other nonempty institutions, by $\mathrm{A} 2$ it suffice to show that some trader wants to switch away from $w_{1}$.

Note that, if $s \in S_{1}$ is such that $p_{s}\left(z_{1}(1)\right)<p_{s}\left(w_{1}\right)$, then $p_{s}\left(z_{1}(1)\right)+\delta<p_{s}\left(w_{1}\right)$ By $\mathrm{A} 1^{*}(\mathrm{i})$, there exists $\underline{r}_{1}\left(p_{s}\left(z_{1}(1)\right)+\delta, p_{s}\left(w_{1}\right)\right)$ such that any buyer at $w_{1}$ who observes a price lower than or equal to $p_{s}\left(z_{1}(1)\right)+\delta$ at institution $z_{1}\left(r_{1}\right)$ with $r_{1} \geq$ $\underline{r}_{1}\left(p_{s}\left(z_{1}(1)\right)+\delta, p_{s}\left(w_{1}\right)\right)$ switches to $z_{1}\left(r_{1}\right)$.

Let $\underline{r}_{1}^{\prime \prime}$ be the maximum among all the $\underline{r}_{1}\left(p_{s}\left(z_{1}(1)\right)+\delta, p_{s}\left(w_{1}\right)\right)$ for profile $s \in S_{1}$ such that $p_{s}\left(z_{1}(1)\right)<p_{s}\left(w_{1}\right)$.

Analogously, if $s \in S_{1}$ is such that $p_{s}\left(z_{1}(1)\right)>p_{s}\left(w_{1}\right)$, then $p_{s}\left(z_{1}(1)\right)-\delta>$ $p_{s}\left(w_{1}\right)$ and there exists $\bar{r}_{1}\left(p_{s}\left(z_{1}(1)\right)-\delta, p_{s}\left(w_{1}\right)\right)$ such that any seller at $w_{1}$ who observes a price higher than or equal to $p_{s}\left(z_{1}(1)\right)-\delta$ at institution $z_{1}\left(r_{1}\right)$ with $r_{1} \leq$ $\bar{r}_{1}\left(p_{s}\left(z_{1}(1)\right)-\delta, p_{s}\left(w_{1}\right)\right)$ switches to $z_{1}\left(r_{1}\right)$. Let $\bar{r}_{1}^{\prime \prime}$ be the minimum among all the $\bar{r}_{1}\left(p_{s}\left(z_{1}(1)\right)-\delta, p_{s}\left(w_{1}\right)\right)$ for profile $s \in S_{1}$ such that $p_{s}\left(z_{1}(1)\right)>p_{s}\left(w_{1}\right)$.

Let $\underline{r}_{1}=\max \left(\underline{r}_{1}^{\prime}, \underline{r}_{1}^{\prime \prime}\right)<1$ and $\bar{r}_{1}=\min \left(\bar{r}_{1}^{\prime}, \bar{r}_{1}^{\prime \prime}\right)>1$ and fi now any $\left.r_{1} \in\right] \underline{r}_{1}, \bar{r}_{1}[$.

Let $s \in S_{1}$. Suppose firs $r_{1}<1$. If $p_{s}\left(z_{1}\left(r_{1}\right)\right) \geq p_{s}\left(w_{1}\right)$, by A1(ii), (weak) sellers at $w_{1}$ want to switch to $z_{1}\left(r_{1}\right)$ with positive probability. Hence, we are left with the case $p_{s}\left(z_{1}\left(r_{1}\right)\right)<p_{s}\left(w_{1}\right)$.

By nondegeneracy, $p_{s}\left(z_{1}(1)\right) \neq p_{s}\left(w_{1}\right)$. By construction, $p_{s}\left(z_{1}\left(r_{1}\right)\right)<p_{s}\left(w_{1}\right)$ implies that $p_{s}\left(z_{1}(1)\right)<p_{s}\left(w_{1}\right)$ and $p_{s}\left(z_{1}\left(r_{1}\right)\right)<p_{s}\left(z_{1}(1)\right)+\delta<p_{s}\left(w_{1}\right)$. Since, by hypothesis, $r_{1} \geq \underline{r}_{1}\left(p_{s}\left(z_{1}(1)\right)+\delta, p_{s}\left(w_{1}\right)\right)$, it follows that buyers switch from $w_{1}$ to $z_{1}\left(r_{1}\right)$.

The case $r_{1}>1$ is analogous. This shows that, as long as both $w_{1}$ and $z_{1}\left(r_{1}\right)$ are nonempty, under Assumption $D$ traders will move from the former to the latter with positive probability.

Suppose now traders are coordinated at state $W$. After an initial mutation where one trader switches to $z_{1}\left(r_{1}\right)$, the argument above shows that there exists a positive 
probability path to the absorbing state where all traders are coordinated at $z_{1}\left(r_{1}\right)$ and $w_{k}$ for $k \neq 1$.

Let now $S_{2}$ be the set of strategy profile $s$ such that $\left.A_{1}(s)=\left\{z_{1}\left(r_{1}\right)\right)\right\}, A_{2}(s)=$ $\left.\left\{w_{2}, z_{2}\right)\right\}$ and $A_{k}(s)=\left\{w_{k}\right\}$ for all $k \geq 3$. An analogous argument shows that there exist $\underline{r}_{2}<1$ and $\bar{r}_{2}>1$ such that, if $z_{2}\left(r_{2}\right)$ is an institution for good 2 with rationing parameter $\left.r_{2} \in\right] \underline{r}_{2}, \bar{r}_{2}$ [, a single mutation from the previous absorbing state suffice to trigger a transition to the absorbing state where all traders are coordinated at $z_{1}\left(r_{1}\right)$, $z_{2}\left(r_{2}\right)$ and $w_{k}$ for $k \geq 3$. The argument proceeds then iteratively.

\section{Example: a simple exchange economy}

We now analyze a simple example in order to show the intuition for the general results. Take an exchange economy with four agents, denoted by $A, A^{\prime}, B$ and $B^{\prime}$. There are two goods, 0 and 1 . All traders are endowed with the same utility functions

$$
U^{i}\left(q_{0}^{i}, q_{1}^{i}\right)=q_{0}^{i} q_{1}^{i}, \quad i=A, B, A^{\prime}, B^{\prime}
$$

with $q_{0}^{i}, q_{1}^{i}$ denoting the quantities $i$ consumes of good 0 and 1 . The initial endowments are given by $e^{A}=e^{A^{\prime}}=\left(e_{0}^{A}, e_{1}^{A}\right)=(3,1)$ and $e^{B}=e^{B^{\prime}}=\left(e_{0}^{B}, e_{1}^{B}\right)=(1,3)$.

Good 0 is traded at a fully competitive market institution without any rationing. It serves as numeraire. All traders have to choose simultaneously and independently the market institution at which each of them wants to trade good 1. A fully competitive institution $w$ is feasible for good 1 , but traders can also choose an alternative constant-rationing institution $z$ with $r_{z}=r>1 .{ }^{9}$ Since traders might opt for different institutions, the price of good 1 need not be the same for all traders. Denote the price at which trader $i$ trades good 1 by $p^{i}$.

Utility maximization gives the following excess demand functions:

$$
\begin{aligned}
& \left(x_{0}^{i}, x_{1}^{i}\right)=\left(\frac{p^{i}-3}{2}, \frac{3-p^{i}}{2 p^{i}}\right), \quad i=A, A^{\prime}, \\
& \left(x_{0}^{j}, x_{1}^{j}\right)=\left(\frac{3 p^{j}-1}{2}, \frac{1-3 p^{j}}{2 p^{j}}\right), \quad j=B, B^{\prime} .
\end{aligned}
$$

Agents learn according to the learning rule described in the example at the end of Sect. 3.1. In the context of our simple exchange economy this means the following. First, an agent never switches to an empty institution. Second, an agent $i$ switches with positive probability from $w$ to the nonempty $z$ if the price and the rationing on $z$ are such that for given price and rationing at $z$ and $w$ agent $i$ would have at least as good off on $z$ than he was on $w$. If the other agent of i's type, denoted by $\widehat{i}$, has already been at $z$, the rule implies that $i$ switches from $w$ to $z$ if $\widehat{i}$ 's utility at $z$ has been larger than i's utility at $w$. A symmetric rule applies of course for the switch from $z$ to $w$.

A state is given by $s=\left(s_{A}, s_{A^{\prime}}, s_{B}, s_{B^{\prime}}\right)$ where $s_{i}$ is the institution for good 1 chosen by trader $i$. Taking into account the possibility of relabeling of the traders, we have to

$\overline{9}$ By symmetry, similar results can be derived in the presence of a constant-rationing institution with $r<1$. 
Table 1 An example

\begin{tabular}{lcccccc}
\hline State & $p_{w}$ & $p_{z}$ & $U^{A}$ & $U^{A^{\prime}}$ & $U^{B}$ & $U^{B^{\prime}}$ \\
\hline$s_{1}=(w w w w)$ & 1 & - & 4 & 4 & 4 & 4 \\
$s_{2}=(z z z z)$ & - & $\frac{1+3 r}{3+r}$ & $\frac{(5+3 r)^{2}}{(1+3 r)(3+r)}$ & $\frac{(5+3 r)^{2}}{(1+3 r)(3+r)}$ & $\frac{(9 r-1)(7+r)^{2}}{(1+3 r)(3+r)}$ & $\frac{(9 r-1)(7+r)^{2}}{(1+3 r)(3+r)}$ \\
$s_{3}=(w z w w)$ & $\frac{5}{7}$ & 3 & $\frac{169}{35}$ & 3 & $\frac{121}{35}$ & $\frac{121}{35}$ \\
$s_{4}=(w w w z)$ & $\frac{7}{5}$ & $\frac{1}{3}$ & $\frac{121}{35}$ & $\frac{121}{35}$ & $\frac{169}{35}$ & 3 \\
$s_{5}=(z z z w)$ & $\frac{1}{3}$ & $\frac{1+6 r}{3+2 r}$ & $\frac{(5+6 r)^{2}}{(3+2 r)(1+6 r)}$ & $\frac{(5+6 r)^{2}}{(3+2 r)(1+6 r)}$ & $\frac{(11+2 r)(18 r-5)}{(3+2 r)(1+6 r)}$ & 3 \\
$s_{6}=(z w z z)$ & 3 & $\frac{2+3 r}{6+r}$ & $\frac{(10+3 r)^{2}}{(6+r)(2+3 r)}$ & 3 & $\frac{(10+r)(2+9 r)}{(6+r)(2+3 r)}$ & $\frac{(10+r)(2+9 r)}{(6+r)(2+3 r)}$ \\
$s_{7}=(w z w z)$ & 1 & $\frac{1+3 r}{3+r}$ & 4 & $\frac{(5+3 r)^{2}}{(1+3 r)(3+r)}$ & 4 & $\frac{(7+r)(9 r-1)}{(1+3 r)(3+r)}$ \\
$s_{8}=(w w z z)$ & 3 & $\frac{1}{3}$ & 3 & 3 & 3 & 3 \\
$s_{9}=(z z w w)$ & $\frac{1}{3}$ & 3 & 3 & 3 & 3 & 3 \\
\hline
\end{tabular}

consider nine different states. The realized utilities of all the states are summarized in Table $1 .{ }^{10}$

We go on by analyzing the absorbing sets of the unperturbed learning processes. Since traders will never switch to an empty institution, it is immediately clear that states $s_{1}$ and $s_{2}$ are absorbing. Furthermore, these are the only absorbing sets. If the process is in state $s_{3}$, agent $A^{\prime}$ will switch to $w$ with strictly positive probability, leading to state $s_{1}$. In state $s_{4}$, agent $B^{\prime}$ will switch to $w$ with strictly positive probability, leading to state $s_{1}$. To see that $s_{5}$ is not an element of an absorbing set, note that for all $r \geq 1, \frac{(11+2 r)(18 r-5)}{(3+2 r)(1+6 r)}>3$. Consequently in state $s_{5}$ agent $B^{\prime}$ will switch to $z$ with strictly positive probability, leading to state $s_{2}$. To see that $s_{5}$ is not an element of an absorbing set, note that for all $r \geq 1, \frac{(10+3 r)^{2}}{(6+r)(2+3 r)}>3$. Consequently in state $s_{6}$ agent $A^{\prime}$ will switch to $z$ with strictly positive probability, leading to state $s_{2}$. Since $\frac{(5+3 r)^{2}}{(1+3 r)(3+r)}<4$, in state $s_{7}$ agent $A^{\prime}$ will switch to $w$ with strictly positive probability. In the resulting state $s_{4}$ agent $B^{\prime}$ will switch to $w$ with strictly positive probability, leading to state $s_{1}$. Finally, in states $s_{8}$ and $s_{9}$ the agents at $z$ will switch with strictly positive probability to the $w$, which results in state $s_{1}$.

Next we show that both states are stochastically stable whenever $r$ is smaller than 22.8. Since there are only two absorbing sets, it suffice to show that the radius of both states is equal, namely one. To see assume that in $s_{1}$ trader $A^{\prime}$ makes a mistake and switches to $z$. Now we are in state $s_{3}$, where the price in the non-market-clearing institution $z$ is 3 , whereas the price in the market clearing institution $w$ is $\frac{5}{7}$. Taking the price and the rationing at $z$ for given, the excess demand of $\operatorname{trader} B$ (and of $B^{\prime}$ ) at $z$ would have been $\left(x_{0}^{B}, x_{1}^{B}\right)=\left(\frac{r+4}{3 r}, \frac{9 r-4}{3 r}\right)$. This would have implied a utility of $U^{B}=\frac{(r+4)(9 r-4)}{3 r^{2}}$. This utility level is higher than the utility agent $B$ has realized at $w, \frac{121}{35}$, provided that $r \in[1,22.8]$. Consequently, in $s_{3}$ agents $B$ and $B^{\prime}$ switch

10 Since the underlying calculations are simple and lengthy we decided to present only the outcomes. The calculations are available from the authors upon request. 
with positive probability from $w$ to $z$, resulting in state $s_{6}$. As we have already seen, the unperturbed learning process implies a strictly positive probability to switch from state $s_{6}$ to state $s_{2}$. So only one mistake is necessary for a positive probability path from state $s_{1}$ to $s_{2}$.

Now assume that in state $s_{2}$ trader $B^{\prime}$ makes a mistake and switches to $w$. In the resulting $s_{5}$ the price in the non-market-clearing institution $z$ is $\frac{1+6 r}{3+2 r}$, whereas the price in the market clearing institution $w$ is $\frac{1}{3}$. Taking the price at $w$ for given, the excess demand of trader $A$ (and of $\left.A^{\prime}\right)$ at $w$ would have been $\left(x_{0}^{A}, x_{1}^{A}\right)=\left(-\frac{4}{3}, 5\right)$. This would have implied a utility of $U^{A}=\frac{25}{3}$. This utility level is higher than the one agent $A$ has realized at $z, \frac{(5+6 r)^{2}}{(3+2 r)(1+6 r)}$, for any level of $r \geq 1$. Consequently, in $s_{5}$ agents $A$ and $A^{\prime}$ switch with positive probability from $z$ to $w$, resulting in state $(z z w z)$. This state is equivalent to $s_{4}$, and as we have already seen, the unperturbed learning process implies a strictly positive probability to switch from state $s_{4}$ to state $s_{1}$. So only one mistake is necessary for a positive probability path from state $s_{2}$ to $s_{1}$.

\section{Extension: agent-specifi rationing}

It is sometimes natural to consider rationing schemes which depend on the characteristics of individual agents, to the effect that different agents are rationed differently within the same market. For instance, rationing might take the form of upper or lower bounds on the agent's net trades ${ }^{11}$ (consider for instance constraints on short-selling in financia markets). Although, for simplicity, we have excluded this possibility in the analysis, it is possible to incorporate this kind of rationing schemes in our framework. In this section, we briefl comment on the necessary changes.

Formally, the uniform rationing function $r_{z}(\cdot)$ of an institution $z$ is replaced with agent-specifi rationing functions $R_{z}^{i}(\cdot)$, which are not define on the set of agents present at $z$, but rather on the (unrationed) excess demand of the agent. Then, the realized excess demand for trader $i$ at institution $z$ is given by

$$
\widetilde{x}_{z}^{i}\left(p^{i}, s\right)=R_{z}^{i}\left(x_{k}^{i}\left(p^{i}\right)\right)
$$

where $z=z(s, i, k) \in Z_{k}$ is such that $i \in N(s, z)$. Assume for consistency that $\left|R_{z}^{i}\left(x_{k}^{i}\left(p^{i}\right)\right)\right| \leq\left|x_{k}^{i}\left(p^{i}\right)\right|$ (traders cannot be forced to buy or sell more than they want) and $\operatorname{sign}\left[R_{z}^{i}\left(x_{k}^{i}\left(p^{i}\right)\right)\right]=\operatorname{sign}\left[x_{k}^{i}\left(p^{i}\right)\right]$ (traders cannot be forced to sell if they want to buy, and vice versa). In particular, $R_{z}^{i}(x)=0$ if and only if $x=0$. As an example, let $K>0$ be an arbitrary upper bound on demand, so that $R_{z}^{i}(x)=\max (x, K)$ (which is still symmetric for all agents) and

$$
\widetilde{x}_{z}^{i}\left(p^{i}, s\right)=\max \left(x_{k}^{i}\left(p^{i}\right), K\right) .
$$

As long as the functions $R_{z}^{i}(\cdot)$ are continuous (as in the example with upper bounds on trade), equilibrium existence results will be unaffected and the model can be analyzed

11 We are grateful to an anonymous referee for suggesting this example. 
as in the main text. Let $p(s)$ denote the equilibrium price (possibly obtained through the use of an appropriate selection) and let

$$
r_{z}^{i}(s)= \begin{cases}\frac{\tilde{x}_{z}^{i}(p, s)}{x_{k}^{i}\left(p^{i}\right)} & \text { if } x_{k}^{i}\left(p^{i}(s)\right)>0 \\ 1 & \text { if } \tilde{x}_{z}^{i}(p(s), s)=x_{k}^{i}\left(p^{i}(s)\right) \\ \frac{x_{k}^{i}\left(p^{i}\right)}{\tilde{x}_{z}^{i}(p, s)} & \text { if } x_{k}^{i}\left(p^{i}(s)\right)<0\end{cases}
$$

which now is, in general, agent-specifi even if the functions $R_{z}^{i}(\cdot)$ were identical for all traders. Impose the additional constraint that either $r_{z}^{i}(s) \leq 1$ for all rationed agents in $z$, or $r_{z}^{i}(s) \geq 1$ for all of them. For instance, an institution might be such that sellers are not rationed $\left(r_{z}^{i}(s)=1\right)$ but buyers are rationed in different degrees $\left(r_{z}^{i}(s)<1\right)$. Hence, if $r_{z}^{i}(s)>1$ (resp. $\left.<1\right)$ for some $i \in N(s, z)$, then $r_{z}^{i}(s) \geq 1$ (resp. $\leq 1$ ) for all $i \in N(s, z)$. This condition is clearly fulfille for the case of upper (or lower) bounds on demand.

How do agents perceive rationing when observing an institution where traders are rationed differently? We consider an aggregate rationing function $r_{z}(s)$ which fulfill the following minimal conditions: (i) $r_{z}(s)=1$ if and only if $r_{z}^{i}(s)=1$ for all $i \in N(s, z)$, (ii) $r_{z}(s)>1$ only if $r_{z}^{i}(s)>1$ for some $i \in N(s, z)$, and (iii) $r_{z}(s)<1$ only if $r_{z}^{i}(s)<1$ for some $i \in N(s, z)$. For instance, $r_{z}(s)$ could be define analogously to $r_{z}^{i}(s)$ but using total excess demands rather than individual ones, or could be based on an arbitrary weighted average of the $r_{z}^{i}(s)$ values.

Rewrite now Assumption A1 as follows:

(A1a) For every strategy profil $s$, every good $k$, and every institution $z \in A_{k}(s)$,

(i) if $i \in N(s, z)$ with $\widetilde{x}_{k}^{i}(s) \geq 0$ and there exists $z^{\prime} \in A_{k}(s)$ with $r_{z^{\prime}}(s) \geq$ $r_{z}^{i}(s)$ (i.e. buyer $i$ is more rationed at $z$ than buyers are in the aggregate at $z^{\prime}$, if at all rationed) and $p_{z^{\prime}}(s) \leq p_{z}(s)$, or

(ii) if $i \in N(s, z)$ with $\widehat{x}_{k}^{i}(s) \leq 0$ and there exists $z^{\prime} \in A_{k}(s)$ with $r_{z^{\prime}}(s) \leq$ $r_{z}^{i}(s)$ (i.e. seller $i$ is more rationed at $z$ than sellers are in the aggregate at $z^{\prime}$, if at all rationed) and $p_{z^{\prime}}(s) \geq p_{z}(s)$,

then agent $i$ leaves institution $z$ with positive probability.

The intuition for A la is identical to the one for A1, except that $r_{z^{\prime}}(s)$ stands for the perception of the magnitude of rationing at institution $z^{\prime}$ (actually, one could make this perception itself agent-specific)

Examination of the proof of Lemma $\mid$ shows that the result holds true in this framework (under Assumption Ala), because still either all buyers are (weakly) rationed or all sellers are. The proof of Theorem 1 is based on Lemma 1 and Assumptions A2 and D, and thus also this result holds true. We conclude that the Walrasian state will remain stochastically stable if we allow for institutions with more complex rationing rules, as, e.g. those where rationing takes the form of upper or lower bounds on agents' net trades. Of course, depending on the economy's characteristics, such institutions themselves could also be part of a stochastically stable state (for a trivial example, consider a very large upper bound which never becomes binding in the relevant range). 


\section{Discussion}

Our results can be interpreted in two ways. On the one hand, coordination on the market-clearing institutions is obtained independently of the characteristics of the alternative available trading institutions. This strong stability result shows that the market-clearing "assumption" is indeed, to a certain extent, justified

On the other hand, some alternative non-market-clearing institutions are also stochastically stable. Hence, nothing guarantees that the actually used trading institutions are efficient - som regulatory interventions might be necessary to improve the functioning of trading institutions. Furthermore, non-market-clearing "stable" institutions can be deliberately designed, if it is in the interest of a market designer.

The aim of this paper has been to show that a proper discussion of the plausibility of assuming market-clearing can be performed within a general equilibrium framework. In order to obtain a tractable model, the analysis has been restricted in three different ways.

First, we consider only pure-exchange economies. Within an economy with production, the firms problems would need to be explicitly tackled. One possibility would be to treat firm as additional agents whose supply function is derived from profi maximization. In a private property economy, this of course raises the issue of whether profi maximization is in the interest of the shareholders. Furthermore, it is unclear how to model learning (i.e. institutional choice) on the side of the firms For the particular case of single-product firms this could be modeled through a myopic behavioral assumption similar to $\mathrm{Al}$.

Second, we have abstracted from equilibrium multiplicity by directly assuming a continuous selection of the Walrasian correspondence to defin the game's payoffs. This raises the question of whether equilibrium multiplicity would have an effect in institutional choice. In order to tackle this question, one might want to superimpose a further learning process within each institution in order to solve the coordination issue.

Third, we have excluded the possibility of multihoming. At any period each trader can trade a specifi good only at one trading institution. If agents can trade a particular good at several institutions at the same time, it is unclear which prices and which allocations would result. Analyzing the impact of multihoming on the choice of the trading platforms is left for future research.

\section{References}

Allen, B.: Continuous Random Selections from the Utility Correspondence. CARESS Working Paper 8525, University of Pennsylvania, Pliladelphia, PA (1985)

Alós-Ferrer, C.: Finite population dynamics and mixed equilibria. Int Game Theory Rev 5(3), 263290 (2003)

Alós-Ferrer, C., Ania, A.B.: The evolutionary stability of perfectly competitive behavior. Econ Theory $26(3), 497-516(2005)$

Alós-Ferrer, C.. Kirchsteiger, G.: Learning and Market Clearing. Mimeo (2008)

Alós-Ferrer, C., Netzer, N.: The Logit-response Dynamics. Mimeo (2007)

Dreze, J.H.: Existence of an exchange equilibrium under price rigidities. Int Econ Rev 16(2), 301-320 (1975) 
Ellison, G.: Basins of attraction, long run stochastic stability, and the speed of step-by-step evolution. Rev Econ Stud 67, 17-45 (2000)

Faias, M., Moreno-García, E, Páscoa, M.R.: Real indeterminacy of equilibria and manipulability. J Math Econ 37, 325-340 (2002)

Feller, W: An Introduction to Probability Theory and Its Applications, vol. I. New York: Wiley (1968)

Gerber, A., Bettzüge, M.O.: Evolutionary choice of markets. Econ Theory 30, 453-472 (2007)

Holt $\mathrm{C}$.: Industrial organization: a survey of laboratory research. In: Kagel, J., Roth, A. Handbook of Experimental Economics, Princeton: Princeton University Press (1995)

Kandori, M., Mailath, G., Rob, R.: Leaming, mutation, and long-run equilibria in games. Econometrica 61, 29-56 (1993)

Karlin, S., Taylor, H.M.: A First Course in Stochastic Processes, vol. 2. San Diego: Academic Press (1975) Mas-Colell, A., Nachbar, J.: On the finitenes of the number of critical equilibria, with an application to random selections. J Math Econ 20,397-409 (1991)

Moreno-Garcia, E.: Strategic equilibria with partially disposable withholdings. Int Game Theory Rev 8, $1-2 !(2006)$

Ockenfels, A., Roth, A.: Last-minute bidding and the rules for ending second-price auctions: evidence from eBay and Amazon on the Internet. Am Econ Rev 92, 1093-1 103 (2002)

Postlewaite, A.: Manipulation via endowments. Rev Econ Stud 46, 255-262 (1979)

Plott, C.: Industrial organization theory and experimental economics. J Econ Lit 20, 1485-1587 (1982)

Schaffer, M.E.: Evolutionarily stable strategies for a finit population and a variable contest size. I Theor Biol 132, 469-478 (1988)

Stahn, H.: Monopolistic behaviors and general equilibrium: a generalization of Nikaido's work. I Math Econ 32, 87-112(1999)

Yi, G.: Manipulation via withholding. Rev Econ Stud 58, 817-820 (1991)

Young, P.: The evolution of conventions. Econometrica 61, 57-84 (1993) 